\title{
Above- and belowground biomass, nutrient and carbon stocks contrasting an open-grown and a shaded coffee plantation
}

\author{
E. L. Dossa - E. C. M. Fernandes · W. S. Reid · \\ K. Ezui
}

Published online: 18 August 2007

(C) Springer Science+Business Media B.V. 2007

\section{Erratum to: Agroforest Syst DOI: $10.1007 / \mathrm{s} 10457-007-9075-4$}

The column headings for the elements in Table 3 had shifted in the original. The corrected Table 3 is shown here on the right.

The online version of the original article can be found under doi:10.1007/s10457-007-9075-4.

E. L. Dossa · E. C. M. Fernandes · W. S. Reid ( $\square)$

Department of Crop and Soil Sciences, Cornell

University, 803 Bradfield Hall, Ithaca, NY 14853, USA

e-mail:wsr1@cornell.edu

K. Ezui

ESA, Université du Bénin, BP 1515, Lome, Togo

Present Address:

E. L. Dossa

Department of Soil and Crop Science, Oregon State

University, Corvallis, OR 97331, USA

Present Address:

E. C. M. Fernandes

ESSD-ARD, The World Bank, 1818 H Street,

Washington, DC 20433, USA
Table 3 Nutrient concentration in biomass components in shaded and open-grown coffee systems

\section{Element (\%)}

\begin{tabular}{|c|c|c|c|c|c|c|}
\hline System and components & & $\mathrm{N}$ & $\mathrm{P}$ & $\mathrm{K}$ & $\mathrm{Ca}$ & $\mathrm{Mg}$ \\
\hline Shaded coffee & & & & & & \\
\hline Coffee $(1)+$ Albizia $(2)$ & & & & & & \\
\hline Leaves & (1) & 3.22 & 0.14 & 1.12 & 1.41 & 0.74 \\
\hline & (2) & 3.60 & 0.19 & 0.80 & 1.24 & 0.52 \\
\hline Twigs & (1) & 1.19 & 0.02 & 0.64 & 0.76 & 0.23 \\
\hline & (2) & 1.29 & 0.06 & 0.50 & 1.01 & 0.22 \\
\hline Branch & (2) & 0.61 & $<0.01$ & 0.36 & 0.36 & 0.06 \\
\hline Stem & (1) & 0.50 & nd & 0.13 & 0.25 & 0.05 \\
\hline & (2) & 0.35 & nd & 0.11 & 0.18 & 0.01 \\
\hline Coarse roots & (1) & 0.79 & nd & 0.20 & 0.28 & 0.04 \\
\hline & (2) & 0.84 & 0.02 & 0.28 & 0.25 & 0.03 \\
\hline Fine roots $2-10 \mathrm{~mm}$ & & 1.82 & 0.03 & 0.29 & 0.72 & 0.10 \\
\hline Fine roots $<2 \mathrm{~mm}$ & & 2.44 & 0.08 & 0.40 & 0.87 & 0.30 \\
\hline Weeds & & 2.85 & 0.16 & 1.7 & 1.55 & 0.77 \\
\hline Litter & & 2.50 & 0.08 & 0.14 & 2.28 & 0.46 \\
\hline Open-grown coffee & & & & & & \\
\hline Coffee & & & & & & \\
\hline Leaves & & 2.47 & 0.15 & 1.42 & 1.05 & 0.62 \\
\hline Twigs & & 0.93 & 0.04 & 0.65 & 0.68 & 0.23 \\
\hline Stem & & 0.40 & nd & 0.16 & 0.30 & 0.07 \\
\hline Coarse root & & 0.45 & nd & 0.19 & 0.46 & 0.06 \\
\hline Fine roots $2-10 \mathrm{~mm}$ & & 0.88 & 0.03 & 0.34 & 1.00 & 0.14 \\
\hline Fine roots $<2 \mathrm{~mm}$ & & 1.23 & 0.09 & 0.41 & 1.11 & 0.58 \\
\hline Weeds & & 1.69 & 0.14 & 1.40 & 1.80 & 1.03 \\
\hline Litter & & 2.09 & 0.09 & 0.08 & 2.01 & 0.65 \\
\hline
\end{tabular}

